Homology, Homotopy and Applications, vol.14(2), 2012, pp.197-209

\title{
CHROMATIC SUBDIVISION OF A SIMPLICIAL COMPLEX
}

\author{
DMITRY N. KOZLOV \\ (communicated by Robert Ghrist)
}

\begin{abstract}
We prove that the protocol complex of the immediate snapshot read/write complex for $n+1$ processors is a simplicial subdivision of the input complex. Our proof is purely geometric, using the Schlegel diagram construction.
\end{abstract}

\section{Introduction}

Recently, simplicial methods have been used by Herlihy, Shavit, Rajsbaum and others, see [HKR, HS], to prove new results in theoretical distributed computing; e.g., see $[\mathbf{A W}]$ for the introduction into that field. In particular, a certain construction for simplicial complexes, called chromatic subdivision, turned out to be central for the analysis of solvability of tasks in the immediate snapshot read/write computational model.

A key property of that construction in the distributed computing context is that a chromatic subdivision of a pseudomanifold is again a pseudomanifold. This has been rigorously verified in the literature; see [HKR, HS]. Furthermore, it has been observed that chromatic subdivision should in fact be a simplicial subdivision of the initial simplicial complex. In this paper we present a rigorous proof of that statement. While it is certainly motivational to know the relevance of the considered spaces and constructions for distributed computing, it is not necessary in order to understand the formulation and the proof of the result. Therefore, we refer to [AW, HKR, HS] for the general distributed computing context and only present here the specific extract that we need.

For the bulk of our argument, we shall limit ourselves to considering the $n$-simplex as the initial complex. The generalization to the case of the arbitrary simplicial complex is straightforward. It will be done in Subsection 4.4 by gluing the chromatic subdivisions of individual simplices together using the colimit construction as the technical language.

For an arbitrary natural number $n$, let $[n]$ denote the set $\{0,1, \ldots, n\}$, and let $\Delta^{n}$ denote the $n$-dimensional simplex. The vertices of $\Delta^{n}$ are indexed by the elements of $[n]$, and, more generally, the $d$-dimensional simplices of $\Delta^{n}$ are indexed by the subsets of $[n]$ of cardinality $d+1$, for all $d=-1, \ldots, n$. The following definition is due to Herlihy and Shavit:

Received September 15, 2011, revised August 22, 2012; published on November 28, 2012.

2000 Mathematics Subject Classification: 57Q05, 68Q85.

Key words and phrases: subdivision, distributed computing, combinatorial algebraic topology, immediate snapshot protocol.

Article available at http://intlpress.com/HHA/v14/n2/a12 and doi:10.4310/HHA.2012.v14.n2.a12

Copyright (C) 2012, International Press. Permission to copy for private use granted. 
Definition 1.1. Let $n$ be a natural number. The simplicial complex $\chi\left(\Delta^{n}\right)$ is a pure $n$-dimensional abstract simplicial complex defined as follows:

1. The vertices of $\chi\left(\Delta^{n}\right)$ are indexed by all pairs $(p, V)$, such that $V \subseteq[n]$, and $p \in V$, so there are $2^{n}(n+1)$ vertices in total.

2. The $n$-dimensional simplices of $\chi\left(\Delta^{n}\right)$ are formed by all sets of vertices

$$
\left\{\left(0, V_{0}\right),\left(1, V_{1}\right), \ldots,\left(n, V_{n}\right)\right\}
$$

satisfying the following axioms:

(i) For all $i, j \in[n]$, we have either $V_{i} \subseteq V_{j}$ or $V_{j} \subseteq V_{i}$;

(ii) For all $i, j \in[n]$, if $i \in V_{j}$, then $V_{i} \subseteq V_{j}$.

The simplicial complex $\chi\left(\Delta^{n}\right)$ is called the chromatic subdivision of $\Delta^{n}$. The intuition behind the terminology is that one can view choosing the first coordinate of the index of each vertex as the vertex coloring of $\chi\left(\Delta^{n}\right)$; this first coordinate can also be interpreted as a process id; see Remark 1.2.
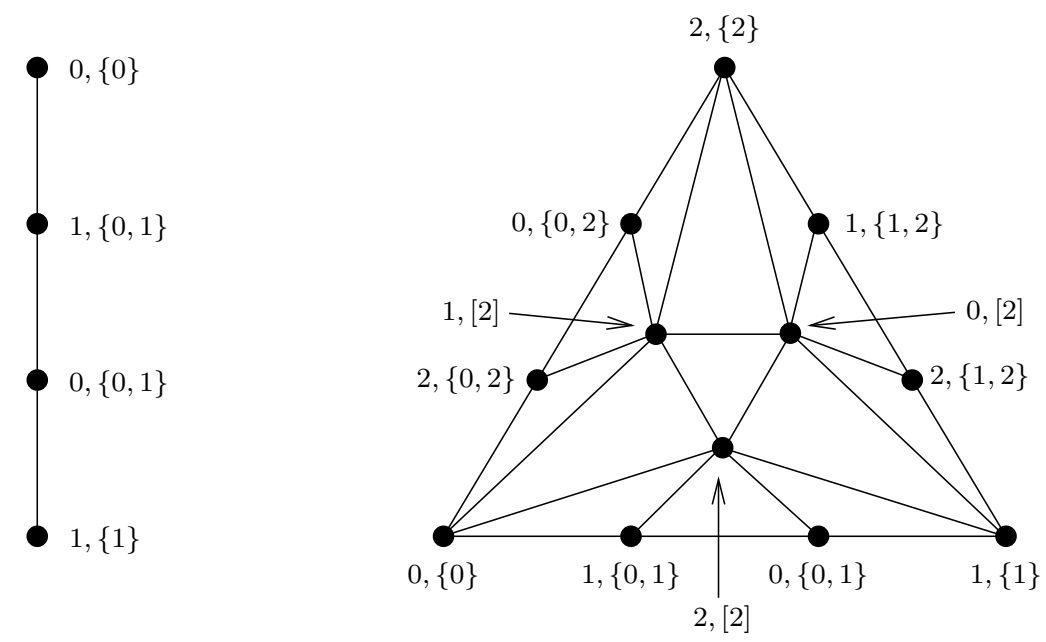

Figure 1: The chromatic subdivisions of 1- and 2-simplex.

Some examples of chromatic subdivisions are shown in Figure 1. Note that condition (ii) in Definition 1.1 can be replaced by the equivalent property: if $V_{i} \nsubseteq V_{j}$, then $i \notin V_{j}$, for all $i, j \in[n]$. By convention, we set $\chi\left(\Delta^{-1}\right)$ to be the empty simplicial complex.

Remark 1.2. The distributed computing interpretation of Definition 1.1 may be motivational. There the vertices are representing views of the execution by individual processors, so a vertex $(p, V)$ stands for "processor number $p$ has view $V$ ". The topdimensional simplices are all possible executions, their vertices should form a compatible set of processor views. So the condition $p \in V$ means "processor $p$ sees itself", the axiom (i) means that for every two processors one of them executed first, and the axiom (ii) means "if processor $j$ sees processor $i$ then it also sees all that $i$ sees". 


\section{Acknowledgements}

I would like to thank Maurice Herlihy and Sergio Rajsbaum for enlightening discussions on theoretical distributed computing. I also benefited greatly from comments and corrections done by Petr Kuznetzov as well as by the anonymous referee. This research has been supported by University of Bremen.

\section{Alternative combinatorial description of the simplicial structure of the chromatic subdivision}

\subsection{Top-dimensional simplices}

We start by rephrasing and analyzing Definition 1.1. First we need another piece of terminology: an ordered set partition of $[n]$ is a tuple of non-empty sets $\left(R_{1}, \ldots, R_{t}\right)$, such that the set $[n]$ is their disjoint union; here $t$ is an integer between 1 and $n+1$.

Proposition 2.1. The $n$-dimensional simplices of $\chi\left(\Delta^{n}\right)$ can be indexed by ordered set partitions of $[n]$. Given an ordered set partition $E=\left(R_{1}, \ldots, R_{t}\right)$, the vertices of the corresponding $n$-simplex $\sigma_{E}$ can be specified as follows: for every $i \in[n]$, set $V_{i}:=$ $R_{1} \cup \cdots \cup R_{j}$, where $j$ is the index such that $i \in R_{j}$, then the pairs $\left(0, V_{0}\right), \ldots,\left(n, V_{n}\right)$ index the vertices of $\sigma_{E}$.

Remark 2.2. The distributed computing interpretation of Proposition 2.1 is straightforward: the ordered set partition $E=\left(R_{1}, \ldots, R_{t}\right)$ encodes the execution consisting of $t$ rounds, where all processes whose id's are in $R_{i}$ execute in round $i$, for all $i=1, \ldots, t$.

Proof of Proposition 2.1. Let $S_{n}$ denote the set of all $(n+1)$-sets of vertices

$$
\left\{\left(0, V_{0}\right), \ldots,\left(n, V_{n}\right)\right\}
$$

of $\chi\left(\Delta^{n}\right)$ satisfying conditions (i) and (ii) of Definition 1.1, and let $P_{n}$ denote the set of all ordered partitions of $[n]$.

Given an ordered set partition, it is straightforward that the corresponding set of vertices described in Proposition 2.1 satisfies conditions (i) and (ii) of Definition 1.1; hence the $n$-simplices encoded by ordered set partitions are actually $n$-simplices of $\chi\left(\Delta^{n}\right)$. This gives us a map $\varphi: P_{n} \rightarrow S_{n}$.

On the other hand, we can also define a map $\psi: S_{n} \rightarrow P_{n}$. Given an $n$-simplex $\sigma=$ $\left\{\left(0, V_{0}\right), \ldots,\left(n, V_{n}\right)\right\}$ of $\chi\left(\Delta^{n}\right)$, we construct the corresponding ordered set partition $\psi(\sigma)$ as follows: Since for all $i, j \in[n]$, we have $V_{i} \subseteq V_{j}$ or $V_{j} \subseteq V_{i}$, there exists a permutation $\pi=(\pi(0), \ldots, \pi(n))$ of the set $[n]$, together with indices $0<i_{1}<i_{2}<\cdots<$ $i_{t-1}<n$, such that $V_{\pi(0)}=V_{\pi(1)}=\cdots=V_{\pi\left(i_{1}\right)} \subsetneq V_{\pi\left(i_{1}+1\right)}=V_{\pi\left(i_{1}+2\right)}=\cdots=V_{\pi\left(i_{2}\right)}$ $\subsetneq \cdots=V_{\pi\left(i_{t-1}\right)} \subsetneq V_{\pi\left(i_{t-1}+1\right)}=\cdots=V_{\pi(n)}$. We set $R_{1}:=\left\{\pi(0), \ldots, \pi\left(i_{1}\right)\right\}, R_{2}:=$ $\left\{\pi\left(i_{1}+1\right), \ldots, \pi\left(i_{2}\right)\right\}, \ldots, R_{t}:=\left\{\pi\left(i_{t-1}+1\right), \ldots, \pi(n)\right\}$. While the permutation $\pi$ is not uniquely determined by the simplex, the ordered set partition $\left(R_{1}, \ldots, R_{t}\right)$ is, and we set $\psi(\sigma):=\left(R_{1}, \ldots, R_{t}\right)$.

Let us show that the composition $\psi \circ \varphi$ is an identity map on $P_{n}$. Given an ordered set partition $E=\left(R_{1}, \cdots, R_{t}\right)$, where $R_{1}=\left\{a_{1}^{1}, \ldots, a_{r_{1}}^{1}\right\}, \ldots, R_{t}=\left\{a_{1}^{t}, \ldots, a_{r_{t}}^{t}\right\}$, we can see that when applying the function $\psi$ to $\varphi(E)$ one can take the permutation $\pi=$ $\left(a_{1}^{1}, \ldots, a_{r_{1}}^{1}, \ldots, a_{1}^{t}, \ldots, a_{r_{t}}^{t}\right)$, and set $i_{1}:=r_{1}-1, i_{2}:=r_{1}+r_{2}-1, \ldots, i_{t-1}:=r_{1}+$ $\cdots+r_{t-1}-1$. This immediately implies that $\psi(\varphi(E))=E$. 
Now let us show that the composition $\varphi \circ \psi$ is an identity map on $S_{n}$. We pick $\sigma=\left\{\left(0, V_{0}\right), \ldots,\left(n, V_{n}\right)\right\} \in S_{n}$, and let $E=\left(R_{1}, \ldots, R_{t}\right)=\psi(\sigma)$, with the permutation $\pi$ and indices $i_{1}, \ldots, i_{t-1}$ chosen as in the definition of the function $\psi$ above. Assume that $\varphi(E)=\left\{\left(0, W_{0}\right), \ldots,\left(n, W_{n}\right)\right\}$; we need to show that $W_{i}=V_{i}$ for all $i$. Unwinding definitions, we see that for all $j$, we have $W_{j}=\left\{i \mid V_{i} \subseteq V_{j}\right\}$, hence we need to verify the identity

$$
\left\{i \mid V_{i} \subseteq V_{j}\right\}=V_{j}
$$

for an arbitrary $j$. To see (1) note that first, since $i \in V_{i}$, the inclusion $V_{i} \subseteq V_{j}$ implies $i \in V_{j}$, hence $\left\{i \mid V_{i} \subseteq V_{j}\right\} \subseteq V_{j}$; on the other hand, if $i \in V_{j}$, then $V_{i} \subseteq V_{j}$, implying $\left\{i \mid V_{i} \subseteq V_{j}\right\} \supseteq V_{j}$.

Altogether, we have shown that the functions $\varphi$ and $\psi$ define a one-to-one correspondence between the sets $S_{n}$ and $R_{n}$.

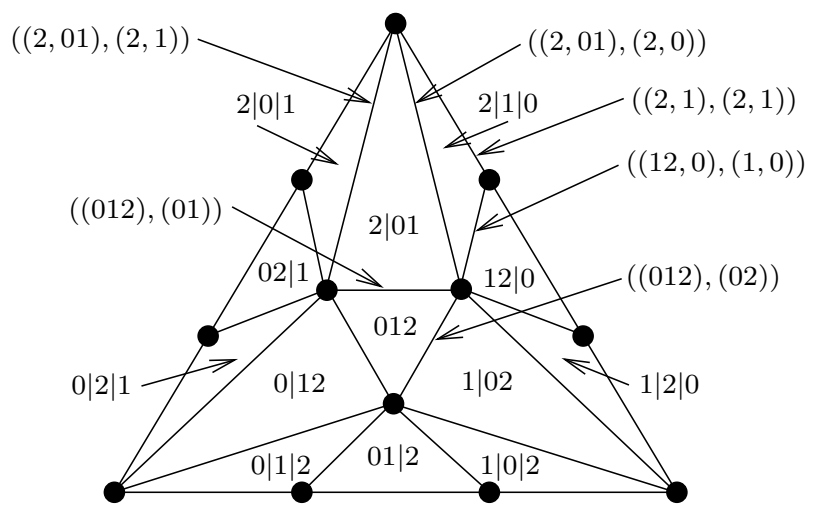

Figure 2: Encoding of all 2-dimensional simplices and of some of the 1-dimensional simplices of $\chi\left(\Delta^{2}\right)$.

In Figure 2 we show the alternative encoding of the top-dimensional simplices of $\chi\left(\Delta^{n}\right)$.

\subsection{Simplices of lower dimension}

It is possible to give a direct description using ordered set partitions for all the simplices of $\chi\left(\Delta^{n}\right)$, not just for those having the maximal dimension. To start with, it is easy to get a description along the lines of Definition 1.1. In fact, a set of vertices $\left\{\left(p_{0}, V_{0}\right), \ldots,\left(p_{d}, V_{d}\right)\right\}$ forms a $d$-simplex if and only if $p_{i} \neq p_{j}$, for all $i \neq j$, and conditions (i) and (ii) of Definition 1.1 are satisfied. This can be seen by the following argument: All the $d$-simplices are obtained by taking a valid $n$-simplex and removing $n-d$ vertices; if this is done, then the conditions above are clearly satisfied. On the other hand, if the set of vertices $S=\left\{\left(p_{0}, V_{0}\right), \ldots,\left(p_{d}, V_{d}\right)\right\}$ satisfies the conditions above, then the set

$$
S \cup\left\{(p,[n]) \mid p \in[n] \backslash\left\{p_{0}, \ldots, p_{d}\right\}\right\}
$$

corresponds to a valid $n$-simplex. Alternatively, using the ordered partition language, we have the following description: 
Proposition 2.3. The simplices of $\chi\left(\Delta^{n}\right)$ are indexed by pairs of tuples:

$$
\tau=\left(\left(B_{1}, \ldots, B_{t}\right),\left(C_{1}, \ldots, C_{t}\right)\right),
$$

such that

1. The sets $B_{1}, \ldots, B_{t}$ are non-empty disjoint subsets of $[n]$.

2. For all $i=1, \ldots, t$, we have $C_{i} \subseteq B_{i}$, and the sets $C_{1}, \ldots, C_{t}$ are also non-empty.

Let $\sigma_{\tau}$ denote the simplex indexed by $\tau$. The dimension of $\sigma_{\tau}$ is $\left|C_{1}\right|+\cdots+\left|C_{t}\right|-1$.

Remark 2.4. The following are the special cases of Proposition 2.3:

1. When $\sigma_{\tau}$ is a vertex, we have $\operatorname{dim} \sigma_{\tau}=0$, i.e., $\left|C_{1}\right|+\cdots+\left|C_{t}\right|=1$, which corresponds to the case $t=1$, and $\left|C_{1}\right|=1$.

2. When $\operatorname{dim} \sigma_{\tau}=n$, we have $\left|C_{1}\right|+\cdots+\left|C_{t}\right|=n+1$, hence $C_{i}=B_{i}$ for all $i=$ $1, \ldots, t$, and $\left(B_{1}, \ldots, B_{t}\right)$ is an ordered partition of $[n]$.

Proof of Proposition 2.3. We shall extend the functions $\varphi$ and $\psi$ from the proof of Proposition 2.1 to the new context. Assume we are given a pair of tuples $\tau=$ $\left(\left(B_{1}, \ldots, B_{t}\right),\left(C_{1}, \ldots, C_{t}\right)\right)$. Let $k=\left|C_{1} \cup \cdots \cup C_{t}\right|$, and let $\left\{p_{1}, \ldots, p_{k}\right\}=C_{1} \cup \cdots \cup$ $C_{t}$. We define

$$
\varphi(\tau):=\left\{\left(p_{1}, V_{1}\right), \ldots,\left(p_{k}, V_{k}\right)\right\},
$$

where the sets $V_{i}$ are constructed using the following rule: for $i=1, \ldots, k$, we let $l(i)$ be the index such that $p_{i} \in C_{l(i)}$, and then set $V_{i}:=B_{1} \cup \cdots \cup B_{l(i)}$. Since $C_{l(i)} \subseteq$ $B_{l(i)}$, we have $p_{i} \in V_{i}$, for all $i$. Also the axioms (i) and (ii) of Definition 1.1 are satisfied, so $\varphi$ is well-defined.

Conversely, assume we are given a $(k-1)$-simplex $\sigma=\left\{\left(p_{1}, V_{1}\right), \ldots,\left(p_{k}, V_{k}\right)\right\}$. After possible reindexing we can choose $0<i_{1}<i_{2}<\cdots<i_{t-1}<n$, such that $V_{1}=$ $V_{2}=\cdots=V_{i_{1}} \subsetneq V_{i_{1}+1}=\cdots=V_{i_{2}} \subsetneq \cdots=V_{i_{t-1}} \subsetneq V_{i_{t-1}+1}=\cdots=V_{k}$. We set $C_{1}:=$ $\left\{p_{1}, \ldots, p_{i_{1}}\right\}, C_{2}:=\left\{p_{i_{1}+1}, \ldots, p_{i_{2}}\right\}, \ldots C_{t}:=\left\{p_{i_{t-1}+1}, \ldots, p_{k}\right\}$, and $B_{1}:=V_{i_{1}}, B_{2}:=$ $V_{i_{2}} \backslash V_{i_{1}}, \ldots, B_{t}:=V_{k} \backslash V_{i_{t-1}}$. Then we define $\psi(\sigma):=\left(\left(B_{1}, \ldots, B_{t}\right),\left(C_{1}, \ldots, C_{t}\right)\right)$. Clearly, the subsets $B_{1}, \ldots, B_{t}$ are disjoint and nonempty, and the subsets $C_{1}, \ldots, C_{t}$ are also nonempty. So to check that $\psi$ is well-defined, we just need to verify that $C_{i} \subseteq B_{i}$. Assume $p_{j} \in C_{l}=\left\{p_{i_{l-1}+1}, \ldots, p_{i_{l}}\right\}$. On one hand, we have $p_{j} \in V_{i_{l}}$, since $p_{j} \in V_{j}$ and $V_{j}=V_{i_{l}}$. On the other hand, we have $p_{j} \notin V_{i_{l-1}}$, since otherwise axiom (ii) of Definition 1.1 would imply that $V_{j} \subseteq V_{i_{l-1}}$, which would contradict the choice of $l$. Thus $p_{j} \in B_{l}$.

Now to see that $\varphi(\psi(\sigma))=\sigma$, for all the simplices $\sigma$, assume that

$$
\sigma=\left\{\left(p_{1}, V_{1}\right), \ldots,\left(p_{k}, V_{k}\right)\right\}
$$

and that the sets $V_{i}$ are already reindexed as necessary for the definition of $\psi(\sigma)$. Assume $\psi(\sigma):=\left(\left(B_{1}, \ldots, B_{t}\right),\left(C_{1}, \ldots, C_{t}\right)\right)$. For an arbitrary $j$, assume that $p_{j} \in$ $C_{l}=\left\{p_{i_{l-1}+1}, \ldots, p_{i_{l}}\right\}$. Then, by construction we have $B_{l}=V_{i_{l}}$. On the other hand, $V_{i_{l}}=V_{j}$, thus $\left(p_{j}, V_{j}\right)$ is a vertex of $\varphi(\psi(\sigma))$. This is true for all $j$, hence $\varphi(\psi(\sigma))=\sigma$.

Finally, assume $\tau=\left(\left(B_{1}, \ldots, B_{t}\right),\left(C_{1}, \ldots, C_{t}\right)\right)$, and let us see that $\psi(\varphi(\tau))=\tau$. Set $i_{1}:=\left|C_{1}\right|, i_{2}:=\left|C_{1} \cup C_{2}\right|, \ldots, i_{t-1}:=\left|C_{1} \cup \cdots \cup C_{t-1}\right|$. Without loss of generality we can assume that $\varphi(\tau)=\left\{\left(p_{1}, V_{1}\right), \ldots,\left(p_{k}, V_{k}\right)\right\}$, with the indexing chosen so 
that $C_{1}=\left\{p_{1}, \ldots, p_{i_{1}}\right\}, C_{2}=\left\{p_{i_{1}+1}, \ldots, p_{i_{2}}\right\}, \ldots, C_{t}=\left\{p_{i_{t-1}-1}, \ldots, p_{k}\right\}$. The construction of $\psi$ specified to this particular set $\left\{\left(p_{1}, V_{1}\right), \ldots,\left(p_{k}, V_{k}\right)\right\}$ yields $\psi(\varphi(\tau))$ $=\tau$.

\subsection{The incidence structure of $\chi\left(\Delta^{n}\right)$}

It is also possible to use the combinatorial description of the simplices from Proposition 2.3 to give an explicit description of the incidence structure of $\chi\left(\Delta^{n}\right)$.

Assume we are given a $d$-simplex $\tau=\left(\left(B_{1}, \ldots, B_{t}\right),\left(C_{1}, \ldots, C_{t}\right)\right)$. The vertices of $\tau$ are indexed by all pairs $(B, c)$, where $c \in C_{i}$, and $B=B_{i}$, for some $1 \leqslant i \leqslant t$. The boundary $\partial \tau$ contains $d+1$ simplices of dimension $d-1$, which can be indexed by the elements of the set $C_{1} \cup \cdots \cup C_{t}$. Specifically, we pick $p \in C_{1} \cup \cdots \cup C_{t}$, and describe a $(d-1)$-simplex $\sigma_{p}$ in $\partial \tau$. Assume $p \in C_{k}$. If $\left|C_{k}\right| \geqslant 2$, then we take

$$
\sigma_{p}=\left(\left(B_{1}, \ldots, B_{t}\right),\left(C_{1}, \ldots, C_{k} \backslash\{p\}, \ldots, C_{t}\right)\right) .
$$

Otherwise, we have $\left|C_{k}\right|=1$, i.e., $C_{k}=\{p\}$. If $k<t$, then we take

$$
\sigma_{p}=\left(\left(B_{1}, \ldots, B_{k-1}, B_{k} \cup B_{k+1}, B_{k+2}, \ldots, B_{t}\right),\left(C_{1}, \ldots, C_{k-1}, C_{k+1}, C_{k+2}, \ldots, C_{t}\right)\right) \text {, }
$$

else $k=t$, and we take

$$
\sigma_{p}=\left(\left(B_{1}, \ldots, B_{t-1}\right),\left(C_{1}, \ldots, C_{t-1}\right)\right) .
$$

Some of the encodings of the lower dimensional simplices are shown on Figure 2, where the reader can also see how the combinatorial incidence structure works.

\section{Preliminaries}

Before proceeding with the main result, we need some preliminaries both from the theory of simplicial complexes as well as from convex geometry.

\subsection{Links, stars, and subdivisions}

Let $K$ be an arbitrary simplicial complex, and let $\sigma$ be any simplex of $K$. We need the following standard notions. The deletion of $\sigma$ is the simplicial subcomplex of $K$ consisting of all simplices which do not contain $\sigma$, i.e.,

$$
\mathrm{dl}_{K}(\sigma)=\{\tau \in K \mid \tau \nsupseteq \sigma\} .
$$

The link of $\sigma$ consists of all the simplices which are disjoint from $\sigma$, but which form a valid simplex together with $\sigma$, i.e.,

$$
\mathrm{lk}_{K}(\sigma)=\{\tau \in K \mid \sigma \cap \tau=\emptyset \text { and } \sigma \cup \tau \in K\} .
$$

Finally, the (closed) star of $\sigma$ is the union of all simplices which form a valid simplex together with $\sigma$, i.e.,

$$
\operatorname{st}_{K}(\sigma)=\{\tau \in K \mid \sigma \cup \tau \in K\} .
$$

The open star is the union of the interiors of all simplices containing $\tau$; it is not a simplicial subcomplex of $K$. 
We have the following equalities:

$$
\begin{gathered}
\operatorname{st}_{K}(\sigma) \cup \mathrm{dl}_{K}(\sigma)=K, \\
\operatorname{st}_{K}(\sigma)=\sigma * \operatorname{lk}_{K}(\sigma),
\end{gathered}
$$

and

$$
\operatorname{st}_{K}(\sigma) \cap \mathrm{dl}_{K}(\sigma)=\{\tau \in K \mid \sigma \cup \tau \in K \text { and } \tau \nsupseteq \sigma\}=\mathrm{lk}_{K}(\sigma) * \partial \sigma,
$$

for all simplices $\sigma \in K$, where $*$ denotes the simplicial join operation. Recall that for abstract simplicial complexes $K$ and $L$ whose sets of vertices are disjoint, the simplicial join is defined as $K * L:=\{\sigma \cup \tau \mid \sigma \in K, \tau \in L\}$. An example is shown in Figure 3. We refer to [Ko07, Subsection 2.1.2] for further examples, and the more detailed description of these concepts.
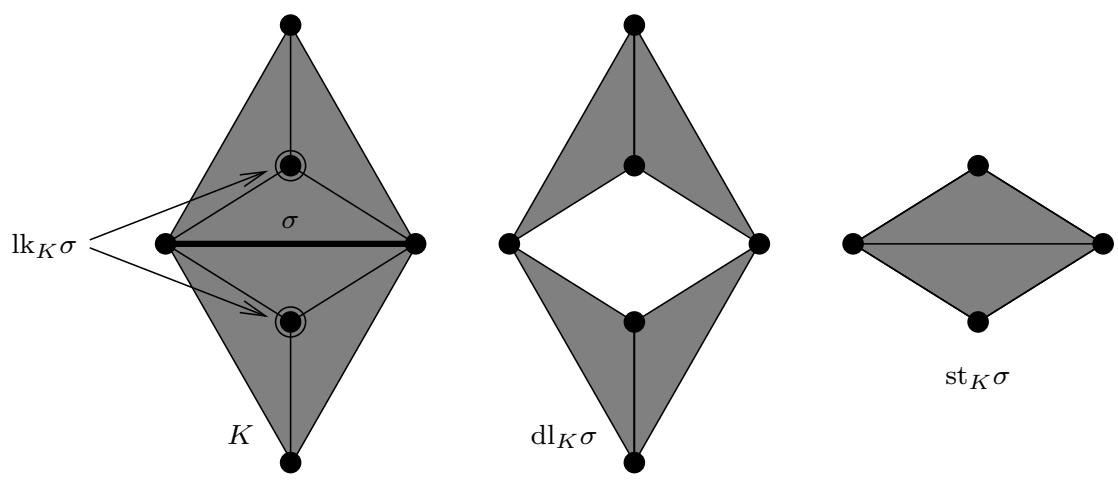

Figure 3: An illustration for link, deletion, and star of a simplex.

Assume now that $X$ is a subdivision of a simplex $\sigma \in K$. Using (2) one sees that the join of $X$ with $\mathrm{lk}_{K}(\sigma)$ is a subdivision of $\mathrm{st}_{K}(\sigma)$; this is a special case of the following general fact:

Fact. Given simplicial complexes $X$ and $Y$, and given $\widetilde{X}$ - a simplicial subdivision of $X$, then the simplicial complex $\widetilde{X} * Y$ is a subdivision of $X * Y$.

One can see this geometrically, if one uses the realization of the join from [Ko07]. Indeed, when $X$ and $Y$ are embedded in complimentary dimensions, and the join is obtained by drawing all the line segments connecting points in $X$ and in $Y$, then it is immediate that replacing $X$ with its subdivision will subdivide the join.

Returning to the subdivision of $\sigma$, assume, in addition, that $X$ only subdivides the interior of $\sigma$, while leaving its boundary $\partial \sigma$ unchanged. Since the extension of the subdivision $X$ to the subdivision of $\operatorname{st}_{K}(\sigma)$ also does not change the link of $\sigma$, we can use (3) to see that this extension of $X$ does not change $\operatorname{st}_{K}(\sigma) \cap \mathrm{dl}_{K}(\sigma)$. This means that we can even further extend the local subdivision $X$ to the entire complex $K$ by leaving $\mathrm{dl}_{K}(\sigma)$ intact in the new subdivision. 


\subsection{Schlegel diagrams}

Recall that a $(d+1)$-dimensional polytope $P$ is a convex hull of finitely many points in $\mathbb{R}^{d+1}$, where we assume that not all points lie on the same affine hyperplane. Clearly, its boundary is homeomorphic to a $d$-sphere. Let $F$ be a $d$-dimensional face on the polytope $P$. By definition, $F$ itself is a $d$-dimensional polytope obtained as an intersection of $P$ with some hyperplane $H$, so that the rest of the polytope lies entirely on one side of this hyperplane. Let $H_{-}$denote the open halfspace bordered by $H$, which does not intersect with the polytope $P$. Choose a point $x$ in $H_{-}$very close to the barycenter of $F$ (in fact any point in the interior of $F$ will do). Now project the boundary of the polytope $P$ along the rays connecting it to $x$ into the hyperplane $H$. If $x$ is sufficiently close to the barycenter of $F$, then the image of that projection will be precisely $F$. Furthermore, by linearity, the images of the faces on the boundary of $P$, excluding $F$ itself, will constitute a polyhedral subdivision of $F$, called the Schlegel diagram of $P$ at $F$, which we shall denote by $\operatorname{Sch}_{F}(P)$. See Figure 4 for examples of Schlegel diagrams of tetrahedra, cube and dodecahedron. More information on Schlegel diagrams can be found in [Gr, HRZ].
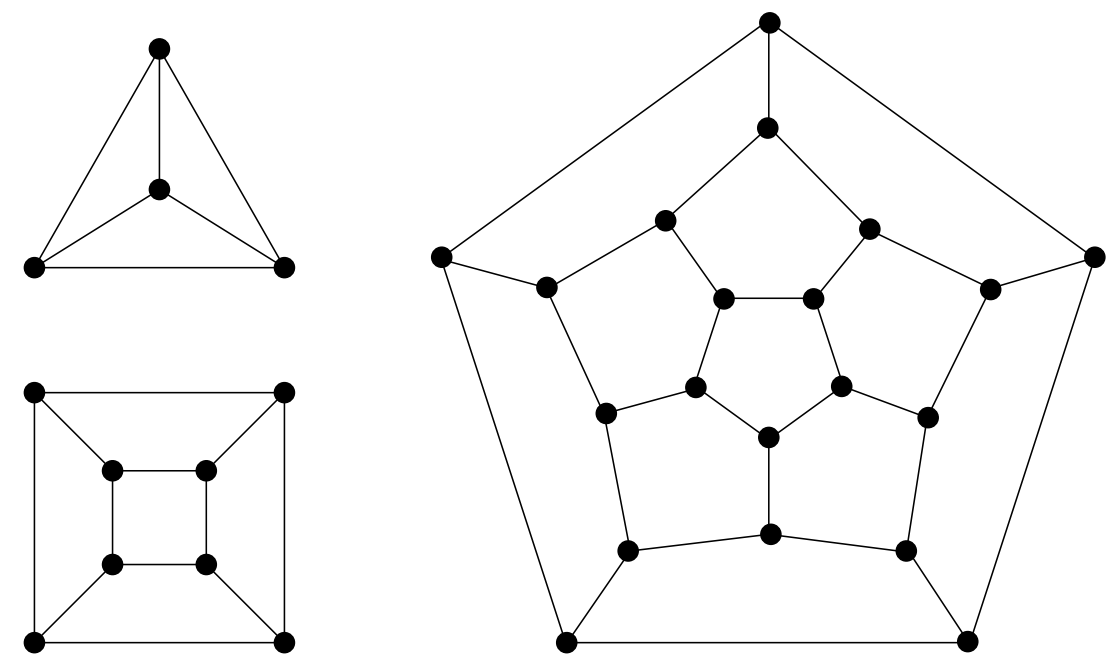

Figure 4: Schlegel diagrams of tetrahedron, cube and dodecahedron.

\section{Main theorem}

\subsection{Subdivisions using Schlegel diagrams of simplicial polytopes}

According to what was said in Section 3, taking a Schlegel diagram of a face in a simplicial polytope provides a simplicial subdivision of the corresponding simplex, which leaves its boundary intact. If this was a simplex in a larger simplicial complex $K$, then this can be extended to a subdivision of the entire complex, as described in Subsection 3.1. All-in-all, given a simplicial complex $K$, its simplex $\sigma$ of dimension $d$, a simplicial polytope $P$ of dimension $d+1$, and an isomorphism $\varphi_{\sigma}$ identifying a face $F$ of $P$ of dimension $d$ with $\sigma$, we let $\Sigma\left(K, \sigma, P, \varphi_{\sigma}\right)$ denote the simplicial subdivision 
of $K$ which is obtained by replacing $\sigma$ with the Schlegel diagram $\operatorname{Sch}_{F}(P)$, and then extending this to the entire $K$.

Assume $\mathcal{F}$ is a set of $d$-simplices of $K$ whose open stars are disjoint, in other words there does not exist a simplex in $K$ which contains two simplices from $\mathcal{F}$. Since only the open star of $\sigma$ gets subdivided, when we pass from the complex $K$ to its subdivision $\Sigma\left(K, \sigma, P, \varphi_{\sigma}\right)$, we can do the corresponding subdivisions simultaneously and independently for each $\sigma \in \mathcal{F}$. We shall denote the total subdivision by $\Sigma(K, \mathcal{F}, P, \Phi)$, where $\Phi=\left\{\varphi_{\sigma} \mid \sigma \in \mathcal{F}\right\}$.

Let now $P$ be a regular simplicial polytope. In this case, the combinatorial type of $\operatorname{Sch}_{F}(P)$ does not depend on the choice of $F$, so we can simply write $\operatorname{Sch}(P)$. Furthermore, since the symmetry group of $P$ acts transitively on the set of flags of $P$, the induced subdivision of $K$ will be independent on the choice of the isomorphism $\varphi$, so we can just write $\Sigma(K, \sigma, P)$.

Assume now that in every dimension $d$ we specified a regular simplicial polytope $P_{d}$ of dimension $d+1$ and call this family of polytopes $\mathcal{P}=\left\{P_{d}\right\}_{d \geqslant 1}$. Assume $K$ is a simplicial polytope of dimension $n$, and let $K^{(i)}$ denote the set of the $i$-dimensional simplices of $K$. Then it is possible to proceed as follows:

\section{Iterated subdivision using Schlegel diagrams of $\mathcal{P}$}

Step 1. Replace $K$ with $X_{1}=\Sigma\left(K, K^{(n)}, P_{n}\right)$.

Step 2. Replace $X_{1}$ with $X_{2}=\Sigma\left(X_{1}, K^{(n-1)}, P_{n-1}\right)$.

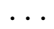

Step i. Replace $X_{i-1}$ with $X_{i}=\Sigma\left(X_{i-1}, K^{(n-i+1)}, P_{n-i+1}\right)$. ...

Step n. Replace $X_{n-1}$ with $X_{n}=\Sigma\left(X_{n-1}, K^{(1)}, P_{1}\right)$.

Finally, we set $\Sigma_{\mathcal{P}}(K):=X_{n}$.

Clearly, $\Sigma_{\mathcal{P}}(K)$ depends heavily on the choice of the family $\mathcal{P}$. There are two classical infinite families of regular simplicial polytopes: the simplices and the crosspolytopes. We can perform a complete analysis for these families.

First, taking the simplex family yields a well-known situation. Indeed, a Schlegel diagram of a simplex is precisely the so-called stellar subdivision. On the other hand, it is well-known that replacing all the top-dimensional simplices with their stellar subdivisions and then proceeding to do so for lower dimensions will yield a barycentric subdivision.

The case of cross-polytopes is more interesting, and we shall connect it to chromatic subdivisions by proving the following result, which will be our main technical statement:

Theorem 4.1. Assume $\mathcal{P}$ is the infinite family of cross-polytopes; then we have $\Sigma_{\mathcal{P}}\left(\Delta^{d}\right)=\chi\left(\Delta^{d}\right)$. In particular, $\chi\left(\Delta^{d}\right)$ is a subdivision of $\Delta^{d}$.

\subsection{Schlegel diagrams of cross-polytopes}

We now return to the framework of Subsection 3.2, though in a special case. If the polytope $P$ is simplicial, then $F$ is a $d$-simplex, and the corresponding Schlegel 
diagram is a simplicial subdivision of $F$. Next, we consider a specific simplicial polytope in $\mathbb{R}^{d+1}$. For $i=1, \ldots, d+1$, let $e_{i}$ denote the point whose $i$ th coordinate is 1 , and all other coordinates are 0 . Let $P_{d}$ be the convex hull of the point set $\left\{e_{1}, \ldots, e_{d+1},-e_{1}, \ldots,-e_{d+1}\right\}$. It is easy to see that these $2 d+2$ points are in convex position and that the obtained polytope is simplicial. This is a geometric realization of the cross-polytope, see e.g., [Cox].

Since $P_{d}$ is a regular polytope, all of its $d$-faces are isomorphic, and for any such isomorphism between two $d$-faces there exists a symmetry of $P_{d}$ extending that isomorphism. In particular, the Schlegel diagram will not depend on which $d$-face of $P_{d}$ we choose, so we just write $\operatorname{Sch}\left(P_{d}\right)$. The examples of Schlegel diagrams for $d=1,2$ are shown in Figure 5 . An alert reader will notice that the boundary complex of $P_{d}$ is precisely the simplicial join of $d+1$ copies of the simplicial complex consisting of two points with no edge between them.

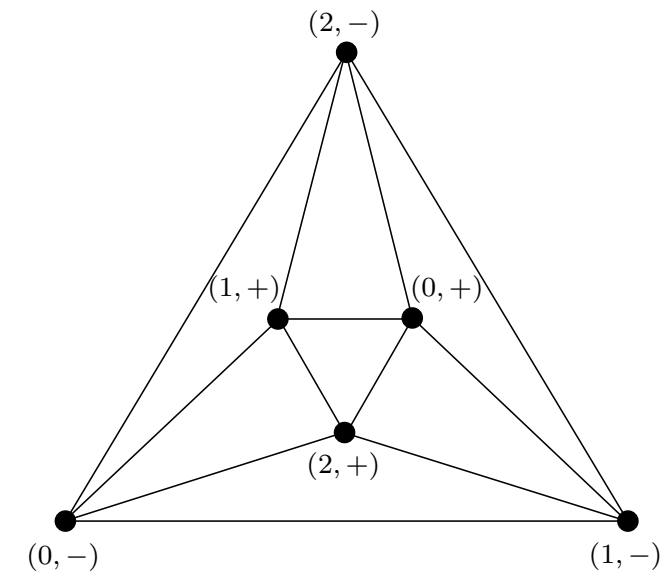

Figure 5: Schlegel diagrams of cross-polytopes for $d=1,2$.

To fix notations, we shall consider the Schlegel diagram associated to the negative $d$-face of $P_{d}$, i.e., the face with vertices $-e_{1}, \ldots,-e_{d+1}$. We now give a combinatorial description of the abstract simplicial complex associated with that Schlegel diagram $\operatorname{Sch}\left(P_{d}\right)$. The set of vertices is indexed by $V=\{(i, s) \mid 0 \leqslant i \leqslant d, s \in\{+,-\}\}$, where for every $i=0, \ldots, d$, the pair $(i,+)$ denotes the inner point of $\operatorname{Sch}\left(P_{d}\right)$ corresponding to the $(i+1)$ th axis, whereas the pair $(i,-)$ denotes the vertex of the $d$-simplex, which we use as the initial face for constructing the Schlegel diagram, corresponding to the $(i+1)$ th axis. Then, the $d$-dimensional simplices of $\operatorname{Sch}\left(P_{d}\right)$ are all tuples $\left(\left(0, s_{0}\right), \ldots,\left(d, s_{d}\right)\right)$, such that $\left(s_{0}, \ldots, s_{d}\right) \neq(-, \ldots,-)$. See Figure 5 for the indexing of simplices in $\operatorname{Sch}\left(P_{2}\right)$.

Clearly, the advantage of using the Schlegel diagrams is that one gets the fact that it is a subdivision of the face for free. We have seen on Figure 5 that $\operatorname{Sch}\left(P_{1}\right)$ is isomorphic to $\chi\left(\Delta^{1}\right)$, whereas $\operatorname{Sch}\left(P_{2}\right)$ is different from $\chi\left(\Delta^{2}\right)$. To get from $\operatorname{Sch}\left(P_{2}\right)$ to $\chi\left(\Delta^{2}\right)$ one needs to further subdivide each of the edges of the triangle and then extend these subdivisions to the subdivision of $\operatorname{Sch}\left(P_{2}\right)$. For higher $d$ one needs to do this several times. 


\subsection{Chromatic subdivision of a simplex}

Let us now perform the iterated subdivision of $\Delta^{d}$ using Schlegel diagrams of $\mathcal{P}$, where $\mathcal{P}$ is the infinite family of cross-polytopes. Set $X_{0}=\Delta^{d}$, let $X_{k}$ as above denote the intermediate steps for $k \geqslant 1$, and let $\Sigma_{\mathcal{P}}\left(\Delta^{d}\right)$ denote the final result. We can give a direct combinatorial description of the simplicial structure which we get at every step of the process.

Proposition 4.2. For $k=0, \ldots, d$, the simplicial complex $X_{k}$ has the following combinatorial description:

1. The vertices of $X_{k}$ are indexed by pairs $(i, A)$, such that $A \subseteq[d], i \in A$, and either $|A|=1$, or $|A| \geqslant d-k+2$.

2. The $d$-simplices of $X_{k}$ are indexed by all sets of $d+1$ vertices corresponding to tuples $\sigma=\left(\left(i_{1}, A_{1}\right), \ldots,\left(i_{d+1}, A_{d+1}\right)\right)$, with $\left\{i_{1}, \ldots, i_{d+1}\right\}=[d]$, satisfying the following conditions:

(i) $\left|A_{1}\right| \leqslant \cdots \leqslant\left|A_{d+1}\right|$,

(ii) $A_{\alpha} \subseteq A_{\beta}$, whenever $\alpha<\beta$, and $\left|A_{\beta}\right| \neq 1$,

(iii) $\left|A_{d-k+2}\right| \geqslant 2$.

Remark 4.3. For $k \geqslant 1$, the transient simplicial complexes $X_{k}$ can also be given a distributed computing interpretation. Namely, we consider all the executions where in the initial stage a certain group of processors, numbering at most $d-k+1$, will only perform the write operation, with the rest of processors functioning normally and performing a write and then the regular immediate snapshot read operation. In particular, the views of those first "write-only" processors consist solely of their own id's. Note that this also interprets the equality $X_{d}=X_{d+1}$, since it does not matter whether the first processor also reads or not after it wrote its id into the shared memory.

Proof of Proposition 4.2. To start with, note that for $k=0$, we only get vertices $(i, A)$, with $|A|=1$, i.e., the vertices $(1,\{1\}), \ldots,(d+1,\{d+1\})$. These form a single $d$-simplex, so our combinatorial description is correct for $X_{0}=\Delta^{d}$.

Let us now show that when passing from $k$ to $k+1$, for $k=0, \ldots, d-1$, the change in the combinatorial simplicial structure described in the proposition corresponds exactly to the transformation from $X_{k}$ to $X_{k+1}$, For convenience, we define $r(\sigma)$ to be the maximal index such that $\left|A_{r(\sigma)}\right|=1$, and set $R(\sigma)=\left\{i_{1}, \ldots, i_{r(\sigma)}\right\}$.

First, we consider the new vertices. For every simplex of $\Delta^{d}$ of codimension $k$, i.e., for every subset $S \subseteq[d]$, such that $|S|=d-k+1$, we add new vertices $\left(s_{1}, S\right), \ldots,\left(s_{d-k+1}, S\right)$, where $S=\left\{s_{1}, \ldots, s_{d-k+1}\right\}$. This is consistent both with the Schlegel construction for cross-polytopes, see Subsection 4.2 , as well as with the description of the set of vertices of $X_{k}$.

Next, we analyze what happens with the $d$-simplices. Many $d$-simplices stay intact. Those which do get subdivided are of the form $\sigma=\left(\left(i_{1}, A_{1}\right), \ldots,\left(i_{d+1}, A_{d+1}\right)\right)$, such that $r(\sigma)=d-k+1$. Each such $\sigma$ gets replaced by new $d$-simplices, which are obtained as follows: Choose a non-empty subset $S \subseteq R(\sigma)$. For simplicity of notations, we can re-index the vertices so that there exists $r$, such that $\left\{i_{1}, \ldots, i_{r}\right\}=R(\sigma) \backslash S$, 
i.e., $\left\{i_{r+1}, \ldots, i_{r(\sigma)}\right\}=S$. Then the new simplex is

$$
\begin{aligned}
\tau=\left(\left(i_{1}, A_{1}\right), \ldots,\left(i_{r}, A_{r}\right),\left(i_{r+1}, R(\sigma)\right), \ldots,\right. & \left(i_{r(\sigma)}, R(\sigma)\right), \\
& \left.\left(i_{r(\sigma)+1}, A_{r(\sigma)+1}\right), \ldots,\left(i_{d+1}, A_{d+1}\right)\right) .
\end{aligned}
$$

We have $r(\tau)=r$, and $R(\tau)=R(\sigma) \backslash S$.

To see that this is exactly what happens when Schlegel diagrams are extended to the entire complex, one can think of the part $\left\{\left(i_{1}, A_{1}\right), \ldots,\left(i_{r(\sigma)}, A_{r(\sigma)}\right)\right\}$ as the maximal face the corresponding cross-polytope indexed by the tuple $(-, \ldots,-)$. In the Schlegel constructions it gets replaced by simplices indexed by all possible nonempty subsets of $\left\{i_{1}, \ldots, i_{r(\sigma)}\right\}$. Then the extension of these subdivisions to the entire complex corresponds to appending this with the rest of the vertices, which is exactly what we do. Clearly, the obtained $d$-simplices are precisely those occurring in our description of the $d$-simplices of $X_{k+1}$, where the order of the vertices is given by construction.

We can now show the result which we announced earlier.

Proof of Theorem 4.1. For $k=d$ we have $d-k+2=2$. This means that there is at most one singleton set among the sets $A_{1}, \ldots, A_{d+1}$. We see that the vertices of $X_{d}$ are all the pairs $(i, A)$, for $i \in[d], i \in A \subseteq[d]$, whereas a set of $d+1$ vertices forms a $d$-simplex if and only if it can be ordered into a tuple $\left(\left(i_{1}, A_{1}\right), \ldots,\left(i_{d+1}, A_{d+1}\right)\right)$ satisfying $\left\{i_{1}, \ldots, i_{d+1}\right\}=[d],\left|A_{1}\right| \leqslant \cdots \leqslant\left|A_{d+1}\right|$, and $A_{\alpha} \subseteq A_{\beta}$, whenever $\alpha<\beta$. In other words, the conditions for the sets of $d+1$ vertices to form $d$-simplices translate precisely into our previous description of the simplicial complex $\chi\left(\Delta^{d}\right)$.

\subsection{Chromatic subdivision of a simplicial complex}

Let $K$ be an arbitrary simplicial complex. Informally, we would like to define $\chi(K)$ to be the simplicial complex obtained from $K$ by replacing each simplex $\sigma \in K$ by $\chi(\sigma)$ simultaneously. This is the same as replacing all the maximal simplices with their chromatic subdivisions and then gluing them together over their boundaries in a coherent way.

While the intuition is clear, one option for a compact rigorous approach is to use the language of colimits; see [Ko07, Section 4.4]. Let $\mathcal{I}(K)$ be the incidence category of $K$ : its objects are indexed by simplices of $K$, and morphisms $\sigma \rightarrow \tau$ correspond to inclusions $\sigma \subseteq \tau$. One can define a functor $\mathcal{S}_{K}: \mathcal{I}(K) \rightarrow \mathbf{S S}$, where $\mathbf{S S}$ is the category of simplicial complexes, by taking $\mathcal{S}_{K}(\sigma)$ to be the subcomplex of $K$ corresponding to $\sigma \in K$ and letting $\mathcal{S}_{K}(\sigma \rightarrow \tau)$ to be the corresponding simplicial inclusion. Passing on to the colimits of diagrams, see [Ko07, Section 15.1], we can say that the colimit of $\mathcal{I}(K)$ is precisely $K$ itself. Indeed, when the maps involved in the diagram are just inclusions, the colimit is precisely what one gets by gluing spaces together in a coherent way.

Definition 4.4. Let $K$ be a simplicial complex, and let the category $\mathcal{I}(K)$ be as above. We construct a functor $\Sigma_{K}: \mathcal{I}(K) \rightarrow$ SS by setting $\Sigma_{K}(\sigma):=\chi(\sigma)$ for $\sigma \in K$, and letting $\Sigma_{K}(\sigma \rightarrow \tau)$ be the corresponding simplicial inclusion. Then we define $\chi(K)$ to be the colimit of $\Sigma_{K}$. 
Note that by construction of $\chi$, if $\sigma \subseteq \tau$, then $\chi(\sigma) \subseteq \chi(\tau)$. Indeed, replacing $[n]$ by any of its subsets in Definition 1.1, will just yield the same object, for a different index set, as all the conditions are preserved. This means that the morphism $\Sigma_{k}(\sigma \rightarrow \tau)$ is well-defined.

Theorem 4.5. For an arbitrary simplicial complex $K$, the complex $\chi(K)$ is a simplicial subdivision of $K$.

Proof of Theorem 4.5. Theorem 4.1 and the construction of $\chi$ imply that composing the functors $\mathcal{S}_{K}$ and $\Sigma_{K}$ with the forgetful functor $\mathbf{S S} \rightarrow$ Top will yield the same diagrams. This means that $K$ and $\chi(K)$ are homeomorphic. Moreover, since each space in the diagram $\Sigma_{K}$ is a subdivision of the corresponding space in the diagram $\mathcal{S}_{K}$, and the simplicial inclusions associated to morphisms $\sigma \rightarrow \tau$ respect these subdivisions, we can conclude that $\chi(K)$ is actually a simplicial subdivision of the complex $K$.

\section{References}

[AW] H. Attiya and J. Welch, Distributed computing: Fundamentals, simulations, and advanced topics, Wiley Series on Parallel and Distributed Computing, 2nd Edition, Wiley-Interscience, Hoboken, NJ, 2004. 432 pp.

[Cox] H.S.M. Coxeter, Regular polytopes, Third edition, Dover Publications, Inc., New York, 1973. xiv+321 pp.

[Gr] B. Grünbaum, Convex polytopes, Second edition, Graduate Texts in Mathematics 221, Springer-Verlag, New York, 2003. xvi+468 pp.

[HRZ] M. Henk, J. Richter-Gebert and G. Ziegler, Basic properties of convex polytopes, in Handbook of discrete and computational geometry, pp. 243-270, CRC Press Ser. Discrete Math. Appl., CRC, Boca Raton, FL, 1997.

[HKR] M. Herlihy, D.N. Kozlov and S. Rajsbaum, Combinatorial topology and distributed computing, to appear.

[HS] M. Herlihy and N. Shavit, The topological structure of asynchronous computability, J. ACM 46 (1999), no. 6, 858-923.

[Ko07] D.N. Kozlov, Combinatorial algebraic topology, Algorithms and Computation in Mathematics 21, Springer-Verlag, New York, 2008, 390 pp. 115 illus.

Dmitry N. Kozlov dfk@math.uni-bremen.de

Department of Mathematics, University of Bremen, 28334 Bremen, Federal Republic of Germany 